\title{
A109 VITAMIN D SUPPRESSES THE PATHOGENIC BEHAVIOUR OF PRIMARY TH17 CELLS FROM PATIENTS WITH EARLY RHEUMATOID ARTHRITIS
}

Jan Piet van Hamburg, ${ }^{1,2}$ Patrick S Asmawidjaja, ${ }^{1,2}$ Nadine Davelaar, ${ }^{1,2}$ Ferry C Cornelissen, ${ }^{1,2}$ Adriana MC Mus, ${ }^{1,2}$ Pieter AGM Bakx, ${ }^{3}$ Edgar M Colin, ${ }^{1}$ Hans van Leeuwen, ${ }^{4}$ JohannaMWHazes, ${ }^{1}$ Radboud JEMDolhain, ${ }^{1}$ ErikLubberts ${ }^{1,2}{ }^{1}$ Department of Rheumatology, Maasstad Hospital, Rotterdam, The Netherlands; ${ }^{2}$ Department of Immunology Maasstad Hospital, Rotterdam, The Netherlands; ' ${ }^{3}$ rasmus MC, University Medical Center and Department of Orthopedics, Maasstad Hospital, Rotterdam, The Netherlands; ${ }^{4}$ Department of Internal medicine, Maasstad Hospital, Rotterdam, The Netherlands

10.1136/ard.2010.148981.12

Background and objectives Recently, the authors showed that CCR6 ${ }^{+}$Th17 cells from early rheumatoid arthritis (RA) patients are potent inducers of a pro-inflammatory feed-back loop upon RA synovial fibroblast (RASF) interaction, including autocrine interleukin (IL)-17A production. In this study, the effect of vitamin D on this pathogenic behaviour of Th17 cells was investigated.

Materials and methods Peripheral CCR6 ${ }^{+}$Th17 cells of patients with early RA, CCR6 ${ }^{+}$Th17/RASF co-cultures and synovial biopsies of patients with established RA, were cultured in the absence or presence of $1,25(\mathrm{OH})_{2} \mathrm{D}_{3}$ the active vitamin D metabolite and/or Etanercept. Intracellular cytokine expression was detected by flow cytometry. Cytokine and matrix metalloprotease (MMP) expression was determined by ELISA. Transcription of factors involved in Th differentiation and function was analysed by quantitative PCR analysis.

Results In Th17 cultures and Th17/RASF co-cultures vitamin D suppressed the expression of IL-17A, IL-22 and IFN- $\gamma$. Furthermore, in Th17/RASF co-cultures, vitamin D induced IL-4 and IL-10 expression, which was accompanied with reduced Ror $\gamma$ t and induced Gata3 transcription. Moreover, vitamin D suppressed IL-6, IL-8, MMP-1 and MMP-3 expression in the Th17/RASF co-cultures and synovial biopsies. Interestingly, the enhanced autocrine production of IL-17A in Th17/RASF co-cultures was inhibited by vitamin D, but not with tumour necrosis factor (TNF)- $\alpha$ neutralisation. Vitamin $\mathrm{D}$ in combination with TNF- $\alpha$ neutralisation has an additive effect on the suppression of Th17 activity as indicated by a significant further reduction in IL-6, IL-8, MMP-1 and MMP-3 expression in the co-cultures and IL- 6 and IL- 8 in the synovial biopsy cultures.

Conclusions These data show that vitamin D modulates the pathogenic behaviour of Th17 cells and may have additional therapeutic potential when used in combination with TNF treatment in RA and potentially other Th17-mediated inflammatory diseases. 